\title{
A CHARACTERIZATION OF NOETHERIAN RINGS BY CYCLIC MODULES
}

\author{
by DINH VAN HUYNH
}

(Received 28 September 1993, revised 25 November 1994)

It is shown that a ring $R$ is right noetherian if and only if every cyclic right $R$-module is injective or a direct sum of a projective module and a noetherian module.

1991 Mathematics subject classification: 16P40, 16D99.

\section{Introduction}

A famous theorem of Hopkins stated that a right artinian ring is right noetherian (see e.g. [1, Theorem 15.20] or [12, Theorem 18.13]). Motivated by this fact, Camillo and Krause raised an interesting question: Is a ring $R$ necessarily right noetherian if for each non-zero right ideal $A$ of $R$, the right $R$-module $R / A$ is artinian? (See [16, Open Problems].) This question still remains unanswered.

By a standard argument, this problem of Camillo and Krause can be reduced to the following form:

$\left(Q_{1}\right)$ Is a domain $D$ necessarily right noetherian if every cyclic right $D$-module is projective or artinian?

From this one might also ask the question:

$\left(Q_{2}\right)$ Is a ring $R$ necessarily right noetherian if every cyclic right $R$-module is injective or noetherian?

On the other hand, Chatters showed in [3, Theorem 3.1] that a ring $R$ is right noetherian if and only if every cyclic right $R$-module is a direct sum of a projective module and a noetherian module. Dually, it is obtained in [7, Theorem 1.1] that a ring $R$ is right artinian if and only if every cyclic right $R$-module is a direct sum of an injective module and a finitely cogenerated module. If we "combine" the assumptions on cyclic modules from these two results for a ring $R$, then $R$ has right Krull dimension as established in [10] (see (1.1) in the next section). However the following question remains open:

$\left(Q_{3}\right)$ Is a ring $R$ necessarily right noetherian if every cyclic right $R$-module is a direct sum of a projective module and a noetherian module or it is a direct sum of an injective module and a finitely cogenerated module?

Clearly $\left(Q_{3}\right)$ includes $\left(Q_{1}\right)$ and $\left(Q_{2}\right)$.

The purpose of this paper is to provide an affirmative answer for a part of $\left(Q_{3}\right)$. Precisely we shall prove the following theorem: 
Theorem. A ring $R$ is right noetherian if and only if every cyclic right $R$-module is injective or a direct sum of a projective module and a noetherian module.

We are unable to derive this result from the above mentioned theorems of [3] and [7], respectively, or from the techniques of their proofs. The following result is an immediate consequence of the theorem and it gives a positive answer to $\left(Q_{2}\right)$ :

Corollary. A ring $R$ is right noetherian (resp. artinian) if and only if every cyclic right $R$-module is injective or noetherian (resp. of finite length).

Concerning this corollary we note that in general, a ring $R$ is not necessarily right noetherian if every cyclic right $R$-module is a direct sum of an injective module and a noetherian module (see [8, Example 11]). Furthermore, we notice that a ring $R$ is right noetherian if every cyclic right $R$-module is a direct sum of a projective module and an injective module ([17, Proposition 2]). Such a ring is called a right CDPI-ring (cf.[18]). CDPI-rings form an interesting class of noetherian rings whose structure has been described completely in the forthcoming paper of J. K. Park and the author "Structure of CDPI-rings".

\section{Preliminaries}

Throughout this paper, rings will mean associative rings with identity and all modules are unitary. For a module $M$ over a ring $R$ we write $M_{R}$ to indicate that $M$ is a right $R$-module. The socle of $M$ is denoted by $\operatorname{Soc}(M)$. If $M=\operatorname{Soc}(M)$, we say that $M$ is a semisimple module. For a ring $R, R=\operatorname{Soc}\left(R_{R}\right)$ if and only if $R$ is right (or left) artinian with zero Jacobson radical. In this case $R$ is called a semisimple ring.

A submodule $N$ of a module $M$ is defined to be an essential submodule of $M$ if $N \cap L \neq 0$ for each non-zero submodule $L$ of $M$. Otherwise $N$ is said to be non-essential. A non-zero module $M$ is called uniform if every non-zero submodule of $M$ is essential in $M$. To say that a module $M$ has finite uniform dimension means that $M$ does not contain an infinite direct sum of non-zero submodules. Every module with Krull dimension and also every factor module of it has finite uniform dimension (for modules and rings with Krull dimension we refer to Godon and Robson [14]).

A module $M$ is defined to be completely injective if every factor module of $M$ is injective.

General background materials can be found in Anderson and Fuller [1], Chatters and Hajarnavis [4], Faith [12], Kasch [15] and Wisbauer [20].

The investigation in this paper is based on the following result which is obtained in [10, Corollary 2.11].

Lemma 1.1. Let $R$ be a ring such that every cyclic right $R$-module is a direct sum of an injective module and a finitely cogenerated module or it is a direct sum of a projective module and a noetherian module. Then $R$ has right Krull dimension. 
The following type of cyclic modules will be used repeatedly in this paper.

A cyclic module $M$ is called a special module (cf. [8]) if the following conditions are satisfied:

(i) $M$ is uniserial, i.e. the set of submodules of $M$ is linearly ordered under inclusion.

(ii) $M$ is non-noetherian but every proper submodule of the maximal submodule $N$ of $M$ is noetherian.

(iii) For any non-zero proper submodule $H$ of $N, M / H$ is injective.

In [8, Example 11] it is shown that there exists a ring which contains an injective special right ideal. However by the same argument as that of proving [8, Lemma 9] we obtain the following result:

Lemma 1.2. For a ring $R, R_{R}$ is not a special module.

For convenience we say that a ring $R$ has property (P) if every cyclic right $R$-module is injective or a direct sum of a projective module and a noetherian module.

In advance to the proof of Theorem we state the following two claims (1.3) and (1.4).

Lemma 1.3. Let $R$ be a ring satisfying (P). Assume that there is a cyclic nonnoetherian indecomposable right $R$-module $M$ such that every cyclic proper submodule of $M$ is noetherian. Then

(a) $M / \operatorname{Soc}(M)$ is a special module,

(b) if $M$ is injective, then $M$ is a special module.

Proof. Let $N$ denote the sum of all noetherian submodules of $M$. Then $M \neq N$ and moreover, $N$ is the unique maximal submodule of $M$, i.e. $M$ is a local module. Let $U$ be a non-zero noetherian submodule of $M$. By $(\mathrm{P}), M / U$ is injective or $M / U=\bar{P} \oplus \bar{H}$ where $\vec{P}_{R}$ is projective and $\bar{H}_{R}$ is noetherian. In the second case, let $H$ denote the inverse image of $\bar{H}$ in $M$. Then $M / H \cong \bar{P}$, implying $M \cong \bar{P} \oplus H$. Since $M$ is indecomposable, we must have $\bar{P}=0$, i.e. $M / U$ is noetherian. However this implies that $M$ is noetherian, a contradiction. Thus $M / U$ is injective. From this we can follow the last part of proving [8, Lemma 6] to see that $M$ is special if $M$ is injective, completing the proof of (b).

Now let $S=\operatorname{Soc}(M)$. By Lemma 1.1 we see that $S_{R}$ is noetherian. Suppose that $S \neq 0$. Then $M / S$ is injective by the previous argument. Put $\bar{M}=M / S$. Then the image $\bar{N}$ of $N$ in $\bar{M}$ is the unique maximal submodule of $\bar{M}$. Since for every noetherian submodule $\bar{T}$ of $\bar{M}, \bar{M} / \bar{T}$ is injective, we can follow the last part of proving [8, Lemma 6] to see that $\bar{M}$ is a special module. This completes the proof of (a) in case $\operatorname{Soc}(M) \neq 0$.

Now consider the case $\operatorname{Soc}(M)=0$. Let $H$ and $K$ be two non-zero finitely generated submodules of $N$. Then $H$ and $K$ are noetherian. If $H \cap K=0$, since $\operatorname{Soc}(M)=0$, we can pick two non-zero elements $x, y$ with $x \in H, y \in K$ such that $x R \neq H, y R \neq K$, and consider the factor module $M /(x R+y R)$. By the above this module is an injective local module. But $M /(x R+y R)$ contains a non-trivial direct sum $H / x R \oplus K / y R$, a contradiction. Hence $I=H \cap K \neq 0$. By the same observation about $M / I$ we obtain $I=H$ or $I=K$, i.e. $H \subseteq K$ or $H \supseteq K$. From this it is easy to see that $M$ is uniserial. Let $L$ be a proper 
submodule of $N$. Then there is an $x \in N$ with $x \notin L$. Hence $x R \supset L$, proving that $L$ is noetherian.

The proof of Lemma 1.3 is complete.

Lemma 1.4. Let $R$ be a ring satisfying (P). If $R_{R}$ is not noetherian and indecomposable, then:

(i) For any cyclic non-noetherian submodule $X$ of $R_{R}, X_{R} \cong R_{R}$.

(ii) If there exists a non-zero noetherian submodule $A$ of $R_{R}$, then $R / A$ is the direct sum of an injective special module and a completely injective noetherian module. Moreover, in this case, there exists a uniform noetherian submodule $U$ of $R_{R}$ such that $R / U$ is special.

Proof. Let $X$ be a cyclic non-noetherian submodule of $R_{R}$. If $X_{R}$ is injective, then $X=R$, since $R_{R}$ is indecomposable. Assume that $X_{R}$ is non-injective. Then by (P), $X_{R}=P \oplus Q$ where $P_{R}$ is a non-zero projective module and $Q_{R}$ is a noetherian module. Since $P_{R}$ is cyclic and $R_{R}$ is indecomposable we must have $P_{R} \cong R_{R}$. This together with the fact that $R_{R}$ has finite uniform dimension (cf. Lemma 1.1) shows that $Q=0$. It follows $X_{R} \cong R_{R}$, proving the statement (i) of Lemma 1.4.

Now assume that $R_{R}$ contains a non-zero noetherian submodule $A$. Then by assumptions about $R, R / A$ can not be a direct sum of a projective module and a noetherian module. Hence $R / A$ is injective by (P). Since, by (1.1), $R / A$ has finite uniform dimension, we have

$$
R / A=X_{1} \oplus \cdots \oplus X_{n},
$$

where each $X_{i}$ is a cyclic injective indecomposable module. It is clear that at least one of the $X_{i}$ 's is not noetherian. We may assume that $X_{1}$ is not noetherian and aim to show first that $X_{1}$ is special.

Let $Y$ be an arbitrary non-zero cyclic proper submodule of $X_{1}$. Clearly, $Y$ is not injective. By (P) and since $X_{1}$ is uniform, it follows that $Y$ is projective or noetherian. Suppose that $Y$ is projective. Since $R_{R}$ is indecomposable, we have $Y_{R} \cong R_{R}$, proving the fact that $R_{R}$ is embedded in $X_{1}$ and hence $R_{R}$ is uniform. Let $u \in R$ such that the image $\bar{u}$ of $u$ in $R / A$ generates $Y$. Then

$$
R_{R} \cong Y_{R}=(u R+A) / A \cong u R /(u R \cap A) .
$$

It follows $u R=L \oplus(u R \cap A)$ where $L$ is a submodule of $u R$ with $L \cong R_{R}$. Since $R_{R}$ is uniform, we must have $u R \cap A=0$. But this is impossible because $u R \neq 0, A \neq 0$ and $R_{R}$ is uniform. Thus $Y_{R}$ can not be projective and hence $Y_{R}$ must be noetherian. Then using Lemma 1.3 for $X_{1}$ we see that $X_{1}$ is a special module.

Let $y \in R$ such that $y+A$ generates $X_{1}$. Then $y R$ has a noetherian submodule $H=y R \cap A$ such that $y R / H$ is special. In particular, $y R$ is not noetherian. Hence $y R \cong R_{R}$ by the statement (i) of Lemma 1.4. It follows that $R_{R}$ contains a noetherian submodule $K$ such that $R / K$ is special. Then $R /(A+K)$ is also a special module, since $(A+K) / K$ is 
a noetherian submodule of $R / K$ and, as we easily see from the definition, factor modules of a special module by its noetherian submodules are again special. But $R /(A+K) \cong(R /$ $A) /((A+K) / A)$, whence $R^{\prime} / K^{\prime}$ is special where $R^{\prime}:=R / A$ and $K^{\prime}$ denotes the image of $K$ in $R^{\prime}$.

On the other hand, from the fact that $X_{1} \cap K^{\prime}$ is noetherian we see that $\left(X_{1}+K^{\prime}\right) / K^{\prime}$ is a special submodule of $R^{\prime} / K^{\prime}$. From the definition of special modules it is easily seen that any special module does not contain proper special submodules. Hence we have $R^{\prime} / K^{\prime}=\left(X_{1}+K^{\prime}\right) / K^{\prime}$, implying $R^{\prime}=X_{1}+K^{\prime}$ and hence $R^{\prime} / X_{1}$ is noetherian. This shows that in (1), $X_{2} \oplus \cdots \oplus X_{n}$ is noetherian. Put $X=X_{2} \oplus \cdots \oplus X_{n}$. Let $Z$ be an arbitrary submodule of $X$ and denote by $C$ the inverse image of $Z$ in $R$. Then $C_{R}$ is noetherian. By the same reason as that for $R / A$ we see that $R / C$ is also injective. This together with $R / C \cong(R / A) /(C / A)=(R / A) / Z \cong X_{1} \oplus(X / Z)$ shows that $X / Z$ is injective. This means that $X$ is completely injective. Since by (1), $R / A=X_{1} \oplus X$, the first statement of (ii) in Lemma 1.4 is therefore verified.

In the above consideration if we choose $A$ to be uniform then $H=y R \cap A$ is also uniform. Since $y R \cong R_{R}, R_{R}$ contains a uniform noetherian submodule $U$ such that $R / U$ is special. This completes the proof of (ii) in Lemma 1.4.

\section{Proof of the theorem}

One direction of the statement is clear. Suppose now that $R$ is a ring satisfying (P). Then every factor ring of $R$ satisfies (P), too. The fact that by Lemma 1.1 a ring satisfying $(P)$ has right Krull dimension will be used repeatedly.

We assume on the contrary that $R$ is not right noetherian.

By Lemma 1.1, $R$ has finite right uniform dimension. Hence

$$
R_{R}=R_{1} \oplus \cdots \oplus R_{k} \oplus R_{k+1} \oplus \cdots R_{n}
$$

where each $R_{i}$ is an indecomposable right $R$-module, $R_{1}, \ldots, R_{k}$ are not noetherian, $R_{k+1}, \ldots, R_{n}$ are noetherian. By assumption we have $k \geqq 1$.

By (P), for each non-zero submodule $U$ of $R_{i}, R_{i} / U$ is noetherian or injective, since each $R_{i}$ is indecomposable. For each $i=1,2, \ldots, k$ there exists a non-zero submodule $U_{i}$ in $R_{i}$ such that $R_{i} / U_{i}$ is not noetherian, hence injective. We fix these $U_{i}, i=1,2, \ldots, k$, and consider factor modules of $R_{j}$ with $j \neq 1$. Let $U$ be an arbitrary submodule of $R_{j}$ with $j \neq 1$. Then by (1)

$$
R /\left(U_{1} \oplus U\right) \cong\left(R_{1} / U_{1}\right) \oplus R_{2} \oplus \cdots \oplus R_{j-1} \oplus\left(R_{j} / U\right) \oplus R_{j+1} \oplus \cdots \oplus R_{n}
$$

From this there exists a submodule $R_{1}^{\prime}$ of $R /\left(U_{1} \oplus U\right)$ which is isomorphic to $R_{1} / U_{1}$. Hence for each proper submodule $C$ of $R_{1}^{\prime}, R_{1}^{\prime} / C$ is not projective, since $R_{1}$ is indecomposable. If $R /\left(U_{1} \oplus U\right)=P \oplus Q$ with projective $P_{R}$ and noetherian $Q_{R}$, we have $R_{1}^{\prime} \nsubseteq Q$ and $I=R_{1}^{\prime} \cap Q$ is noetherian. Hence $R_{1}^{\prime} / I$ is not noetherian, therefore it is injective. On the other hand, it is easy to see that $R_{1}^{\prime} / I$ can be embedded isomorphically in $P$. It follows that $R_{1}^{\prime} / I$ is a non-zero projective module, a contradiction. Hence, by 
(P), $R /\left(U_{1} \oplus U\right)$ must be injective. Hence by (2) we see that $R_{2}, \ldots, R_{j-1}, R_{j} / U, R_{j+1}, \ldots, R_{n}$ are injective. In other words, every $R_{j}$ for $j \neq 1$ is completely injective.

Case 1: $k \geqq 2$. Using the same argument for $R /\left(U_{2} \oplus U^{\prime}\right)$, being isomorphic to $\left(R_{1} / U^{\prime}\right) \oplus\left(R_{2} / U_{2}\right) \oplus R_{3} \oplus \cdots \oplus R_{n}$, where $U^{\prime}$ is a submodule of $R_{1}$ we see that $R_{1} / U^{\prime}$ is injective. Combining this with the above facts we see that $R_{i}$, for $i=1,2, \ldots, n$, is completely injective. Now let $M$ be an arbitrary maximal submodule of $R_{R}$. Then there exists an $R_{i}$ in (1) such that $R_{i} \nsubseteq M$. Hence $R / M=\left(R_{i}+M\right) / M \cong R_{i} /\left(R_{i} \cap M\right)$ and so $R / M$ is injective. This shows that every simple right $R$-module is injective, i.e. by definition, $R$ is a right V-ring. Since $R$ has right Krull dimension, $R$ is then right noetherian by [2]. This is a contradiction to our assumption.

Case 2: $k=1$. First we have that $R_{1}$ is not noetherian and, as shown above, that the noetherian modules $R_{2}, R_{3}, \ldots, R_{n}$ are all completely injective. However, in this case, $R_{1}$ may be not injective. For each $0 \neq x \in R_{1}$, consider the $R$-homomorphism $\phi_{j}: R_{j} \rightarrow R_{1}$ defined by $\phi_{j}\left(r_{j}\right)=x r_{j}\left(r_{j} \in R_{j}, 2,3, \ldots, n\right)$. Then $R_{j} / k e r \phi_{j}$ is isomorphic to a proper submodule of $R_{1}$. Since $R_{j} / \operatorname{ker} \phi_{j}$ is injective, we must have $R_{j}=\operatorname{ker} \phi_{j}$, i.e. $x R_{j}=0$. This shows

$$
R_{1}\left(R_{2} \oplus \cdots \oplus R_{n}\right)=0
$$

or equivalently, $R_{2} \oplus \cdots \oplus R_{n}$ is an ideal of $R$. Hence we may restrict ourself on the factor ring $R /\left(R_{2} \oplus \cdots \oplus R_{n}\right)$ which is $R$-isomorphic to $R_{1}$. This means, from now on we may assume that $R$ is a ring satisfying (P), $R_{R}$ is indecomposable but not noetherian. Hence we have Lemma 1.4 at our disposal.

(a) Assume that there exists a non-zero element $a$ of $R$ such that $a R$ is noetherian. By the last part of statement (ii) of Lemma 1.4, the uniform dimension $d$ of $R_{R}$ is at most 2 .

Next we show that $d=1$, i.e. $R_{R}$ is uniform. For this purpose we first verify that the right socle $S$ of $R$ is zero. Suppose that $S \neq 0$. Then $S_{R}$ is noetherian by Lemma 1.1 . From this and Lemma 1.4, $R / S$ is a right self-injective ring such that

$$
R / S=\bar{D} \oplus \bar{B}
$$

where $\bar{D}_{R}$ is a special module and $\bar{B}_{R}$ is a completely injective noetherian module. For an arbitrary non-zero element $\bar{d}$ of $\bar{D}$ consider the homomorphism $\phi$ from $\bar{B}_{R}$ to $\bar{D}_{R}$ defined by $\phi(\bar{b})=\bar{d} . \bar{b}$ for all $\bar{b} \in \bar{B}$. If $\bar{d} . \bar{B}$ is non-zero, then $\bar{d} . \bar{B}$ is a non-zero injective noetherian submodule of $\bar{D}$, since $\bar{d} . \bar{B} \cong \bar{B} / \operatorname{ker} \phi$. This is a contradiction to the fact that $\bar{D}_{R}$ is special. Hence $\bar{d} \cdot \bar{B}=0$. It follows that $\bar{D} \cdot \bar{B}=0$, or equivalently, $\bar{B}$ is a (two-sided) ideal of $R / S$. Since $(R / S) / \bar{B} \cong \bar{D}_{R},(R / S) / \bar{B}$ is a special module, a contradiction to Lemma 1.2. Thus the right socle of $R_{R}$ must be zero.

Assume now that $d=2$. By Lemma $1.4, R_{R}$ contains a uniform noetherian submodule $U$ such that $R / U$ is special. Let $V$ denote a complement of $U$ in $R_{R}$. Then by assumption, $V \neq 0$ and since $V_{R}$ is embedded in $R / U, V_{R}$ contains a non-zero noetherian submodule $V_{1}$. By Lemma 1.4 we have 


$$
R / V_{1}=\bar{S} \oplus \bar{T}
$$

where $\bar{S}$ is special, injective and $\bar{T}$ is noetherian, completely injective. Since the right socle of $R$ is zero, we may assume $V_{1} \neq V$, and therefore the right $R$-module $R / V_{1}$ has uniform dimension at least 2 . This shows that in (3), $\bar{T}$ is non-zero. Let $\bar{U}$ denote the image of $U$ in $R / V_{1}$ and $\widetilde{V}_{1}$ the image of $V_{1}$ in $R / U$. Since $\widetilde{V}_{1}$ is noetherian and

$$
\left(R / V_{1}\right) / \bar{U} \cong R /\left(U+V_{1}\right) \cong(R / U) / \tilde{V}_{1},
$$

$\left(R / V_{1}\right) \bar{U}$ must be special. Since $(\bar{T}+\bar{U}) / \bar{U} \cong \bar{T} /(\bar{T} \cap \bar{U})$, and $\bar{T}$ is completely injective, it follows that $(\bar{T}+\bar{U}) / \bar{U}$ is an injective noetherian submodule of the special module $\left(R / V_{1}\right) / \bar{U}$. Then by the properties of special modules we must have $\bar{T}+\bar{U} \subseteq \bar{U}$, or equivalently, $\bar{T} \subseteq \bar{U}$. Since $\bar{U}_{R}$ is uniform, we must have $\bar{U}=\bar{T}$. But $\bar{U} \cong U, U_{R}$ has to be injective. This is a contradiction since $R_{R}$ is indecomposable.

Thus $d=1$, i.e. $R_{R}$ is uniform. If the prime radical $N$ of $R$ is zero, then $R$ is semiprime and since $R$ has right Krull dimension, $R$ is right Goldie (see [14]). It follows that $R$ is a right Ore domain, in particular, $R$ does not contain non-zero zerodivisors. On the other hand, by assumption, $R_{R}$ contains a non-zero cyclic noetherian submodule $a R$ for some $a$ in $R$. Since $a R \cong R / r(a)$ where $r(a)=\{s \in R$, as $=0\}=0$, it follows that $R$ is right noetherian, a contradiction.

Thus we must have $N \neq 0$. In this case the Jacobson radical $J$ of $R$ is non-zero. Recall that $J$ is the intersection of all maximal submodules of $R_{R}$. Since $R_{R}$ is uniform and $R_{R}$ contains a non-zero noetherian submodule, $J_{R}$ contains a non-zero noetherian submodule $H$, too. Then by Lemma 1.4 we have

$$
R / H=S^{\prime} \oplus T^{\prime}
$$

where $S_{R}^{\prime}$ is special and $T_{R}^{\prime}$ is noetherian. Since $H \subseteq J$ we easily see that $J / H$ is also the intersection of all maximal submodules of $R / H$, i.e. $J / H$ is the Jacobson radical of the right $R$-module $R / H$. Therefore $J / H=\left((J / H) \cap S^{\prime}\right) \oplus\left((J / H) \cap T^{\prime}\right)$. Hence every finitely generated submodule of $J_{R}$ is noetherian and $(J / H) \cap S^{\prime}$ is exactly the maximal submodule of the special module $S^{\prime}$. Let $X$ be a cyclic submodule of $R_{R}$ such that $(X+H) / H=S^{\prime}$. Then $X_{1}=X \cap J$ is not noetherian and $X / X_{1}$ is simple. Let $Y$ be an arbitrary non-zero cyclic submodule of $X_{1}$. Then $Y_{R}$ is noetherian. By Lemma 1.4, we have $X \cong R_{R}$ and, if we denote the factor module $X / Y$ by $\bar{X}$, then

$$
\bar{X}=\bar{H} \oplus \bar{K},
$$

where $\bar{H}_{R}$ is injective, special and $\bar{K}_{R}$ is injective, noetherian. Let $K$ be the inverse image of $\bar{K}$ in $X$. Then $K_{R}$ is noetherian. If $K \nsubseteq X_{1}$, then $K+X_{1}=X$, implying the fact that $X_{1} /\left(X_{1} \cap K\right)$ is cyclic. Since $X_{1} \cap K$ is noetherian, in particular finitely generated, it follows that $X_{1}$ is finitely generated, too. This together with $X_{1} \subseteq J$ shows that $X_{1}$ is noetherian, a contradiction. Hence $K \subset X_{1}$. If $K \nsubseteq Y$ then, since $K / Y(=\bar{K})$ is injective, $K / Y$ is a non-zero direct summand of $R / Y$, i.e. there exists a right ideal $L$ of $R$ such that 
$L \supseteq Y$ and $R / Y=K / Y \oplus L / Y$. From this there is a maximal right ideal $L^{\prime}$ of $R$ with $L^{\prime} \nsupseteq K$, a contradiction to $K \subset X_{1} \subseteq J$. Hence $K \subseteq Y$, or equivalently, $\bar{K}$ is zero, i.e. $X / Y$ is a special module. Thus we have shown that for each non-zero cyclic submodule $Y$ of $X_{1}, X / Y$ is special. From this and since $X_{R}$ is uniform it is easy to see that every cyclic proper submodule of $X$ is noetherian. By Lemma 1.3, $X_{R}$ or $X / \operatorname{Soc}\left(X_{R}\right)$ is special. But $\operatorname{Soc}\left(X_{R}\right)=0$, since we have shown above that $\operatorname{Soc}\left(R_{R}\right)=0$. Hence $R_{R}$, being isomorphic to $X_{R}$, is a special module, a contradiction to Lemma 1.2. This last contradiction shows that (a) cannot happen. It remains to consider the final possibility:

(b) For each $0 \neq x \in R, x R$ is not noetherian. Then, by Lemma 1.4 we must have

$$
x R \cong R_{R} \text { for all } 0 \neq x \in R .
$$

From this and from the fact that $R_{R}$ is indecomposable it follows that $R$ has no non-zero zerodivisors. Since $R$ has right Krull dimension, $R$ is then a right Ore domain. In particular, $R_{R}$ is uniform.

Let $M$ be an arbitrary maximal submodule of $R_{R}$. We aim to show that $R / M$ is an injective right $R$-module. Clearly, there is a non-zero cyclic submodule $y R$ of $M$ such that $R / y R$ is not noetherian. Moreover, since $R_{R}$ is uniform, $R / y R$ can not be decomposed into a direct sum of a projective module and a noetherian module. Hence by (P), $R / y R$ is injective. We have

$$
R / y R=I_{1} \oplus \cdots \oplus I_{n}
$$

where each $I_{i}(i=1, \ldots, n)$ is an indecomposable injective module. We may, without loss of generality, assume that $I_{1}$ is not noetherian. Since $R_{R}$ is uniform and $I_{1}$ is indecomposable it is easy to see that each cyclic proper non-zero submodule of $I_{1}$ is neither injective nor projective, it follows from this and $(\mathrm{P})$ that such a submodule of $I_{1}$ is noetherian. By Lemma 1.3, $I_{1}$ is a special module. Since $R_{R}$ is uniform, $I_{1}$ is not projective.

Now, since $y R \cong R_{R}$ there exists a non-zero submodule $V$ of $y R$ such that $y R / V$ is not noetherian. Using the same argument as for $R / y R$ we see that $y R / V$ is injective and contains an injective special submodule, say $J_{1}$. We have

$$
R / V=y R / V \oplus T
$$

for some submodule $T$ of $R / V$. Moreover, by (4) it holds that $R / y R \cong T$. Hence $T$ is injective and contains an injective special submodule which we denote by $J_{2}$. Thus $R / V$ is injective and we have

$$
R / V=J_{1} \oplus J_{2} \oplus J_{3} \oplus \cdots \oplus J_{h}
$$

where each $J_{i}$ is injective indecomposable, $J_{1}, J_{2}$ are special modules. Let $M_{i}$ be a maximal submodule of $J_{i}$ for each $i=1,2, \ldots, h$. Then for each $i \neq 1$ we have

$$
(R / V) / M_{i} \cong J_{1} \oplus J_{2} \oplus \cdots \oplus J_{i-1} \oplus\left(J_{i} / M_{i}\right) \oplus J_{i+1} \oplus \cdots \oplus J_{h}
$$


Since $J_{1}$ is neither noetherian nor projective, so is $(R / V) / M_{i}$. By $(\mathrm{P})$ and since $R_{R}$ is uniform, $(R / V) / M_{i}$ must be injective. Hence $J_{i} / M_{i}$ is injective for every $i \neq 1$. Furthermore, since $J_{2}$ is also not noetherian, not projective, by the same observation for $(R / V) / M_{1} \cong\left(J_{1} / M_{1}\right) \oplus J_{2} \oplus J_{3} \oplus \cdots \oplus J_{h}$, we obtain that $J_{1} / M_{1}$ is also injective. From these facts it is easy to see that for each maximal submodule $H$ of $R / V$ the factor module $(R / V) / H$ is injective. Since $M$ was a maximal submodule of $R_{R}$ containing $V$, it follows that $R / M$ is injective as desired.

In fact we have shown that $R$ is a right $V$-ring, hence $R$ is right noetherian as concluded in Case 1. This however is a contradiction to our assumption.

The proof of the theorem is complete.

\section{Some remarks}

Let $R$ be a ring such that every cyclic right $R$-module is a direct sum of an injective module and a noetherian module. Then by [9, Theorem 7] $R$ has right Krull dimension and if $R$ is semiprime, $R$ is right noetherian by [9, Proposition 8]. In general, such a ring needs not be right notherian as is shown in [8, Example 11]. Example 11 of [8] shows also that there exists a cyclic injective and projective module $M$ (over a ring $R$ ) such that cyclic submodules of any factor module of $M$ are injective or noetherian while $M$ is not noetherian. This shows that in general the statement in our theorem cannot be carried over to modules. However, Chatters' theorem in [3] can be transferred to modules as obtained in [19]: A finitely generated module $M$ is noetherian if every factor module of $M$ is a direct sum of an $M$-projective module and a noetherian module.

C. Faith called a ring $R$ a right PCI ring if every cyclic right $R$-module is injective or isomorphic to $R_{R}$. By his result in [11] and a result of Damiano in [6] such a ring is either semisimple or it is a non-artinian, right hereditary, right noetherian, simple domain. The latter ring is usually called a right PCI domain. The existence of right PCI domains is established in [5].

Rings $R$ each of whose cyclic right $R$-modules is injective or projective have been studied in [13] and [18]. It is shown that such a ring is a ring-theoretic direct sum of a semisimple ring and a right PCI domain.

In [18], rings $R$ with the property that every cyclic right $R$-module is a direct sum of an injective module and a projective module have been investigated. However it is shown just recently in [17] that such a ring is right noetherian. Moreover a significant result has been obtained in [17] which says that a cyclic module $M$ has finite uniform dimension if each cyclic submodule of any factor module of $M$ is CS. This result provides a number of applications in the area. In particular, it motivates the investigation in [10] in which the statement of Lemma 1.1 is obtained. Without Lemma 1.1 it is impossible for us to establish the theorem in this paper.

Acknowledgements. This investigation was carried out when the author visited the Departments of Mathematics of the Albert-Ludwigs-University in Freiburg and the University of Murcia. He wishes to express his sincere thanks to Professor O. H. Kegel 
(Freiburg), Professor J. L. Gómez Pardo (Murcia) and the members of both institutions for invitations with kind support and hospitality.

\section{REFERENCES}

1. F. W. Anderson and K. R. Fuller, Rings and Categories of Modules (Springer-Verlag 1974).

2. A. K. Boyle and K. R. Goodearl, Rings over which certain modules are injective, Pacific J. Math. 58 (1975), 43-53.

3. A. W. Chatters, A characterization of right noetherian rings, Quart. J. Math. Oxford (2) 33 (1982), 65-69.

4. A. W. Chatters and C. R. Hajarnavis, Rings with Chain Conditions (Pitman, London 1980).

5. J. H. Cozzens, Homological properties of the ring of differential polynomials, Bull. Amer. Math. Soc. 76 (1970), 75-79.

6. R. F. Damiano, A right PCI ring is right noetherian, Proc. Amer. Math. Soc. 77 (1979), 11-14.

7. Dinh van Huynh and Nguyen V. Dung, A characterization of artinian rings, Glasgow Math. J. 30 (1988), 67-73.

8. Dinh van Huynh and P. F. Smith, Some rings characterised by their modules, Comm. Algebra (6) 18 (1990), 1971-1988.

9. Dinh van Huynh, Nguyen V. Dung and P. F. Smith, A characterization of rings with Krull dimension, J. Algebra 132 (1990), 104-112.

10. Dinh van Huynh, Nguyen V. Dung and R. Wisbauer, On modules with finite uniform and Krull dimension, Arch. Math. 57 (1991), 122-132.

11. C. FaITH, When are proper cyclics injective?, Pacific J. Math. 45 (1973), 97-112.

12. C. FAITH, Algebra II: Ring Theory (Springer-Verlag 1976).

13. V. K. GoEL, S. K. JAIN and S. SINGH, Rings whose cyclic modules are injective or projective, Proc. Amer. Math. Soc. 53 (1975), 16-18.

14. R. Gordon and J. C. Robson, Krull dimension (Mem. Amer. Math. Soc. 123, 1973).

15. F. KASCH, Moduln und Ringe (Teubner, Stuttgart 1977).

16. A. Kertesz (Editor), Associative Rings, Modules and Radicals, in Proc. Colloquium at Keszthely in 1971 (J. Bolyai Math. Soc. North-Holland Publ. Co., Amsterdam-London-Budapest, 1973).

17. B. L. Osofsky and P. F. Smith, Cyclic modules whose quotients have complements direct summands, J. Algebra 139 (1991), 342-354.

18. P. F. SMith, Rings characterized by their cyclic modules, Canad. J. Math. 24 (1979), 93-111.

19. P. F. SMith, Dinh VAN Huynh and NGuyen V. Dung, A characterization of noetherian modules, Quart. J. Math. Oxford 41 (1990), 225-235.

20. R. Wisbauer, Foundations of Module and Ring Theory (Gordon and Breach, Reading 1991).

Institute of Mathematics

P.O. Box 631 Воно

Hanol, Vietnam 